\title{
Ammonia Oxidizing Bacteria in Industrial Waste Water
}

\author{
Shah M \\ Industrial Waste Water Research Lab \\ Division of Applied \& Environmental Microbiology \\ India
}

\begin{abstract}
Denaturing Gradient Gel Electrophoresis is a influential and feasible device for sequence examination of the variety of complicted natural microbial populations. For PCR amplification of $16 S$ rDNA products and specific primers were used and analyzed by denaturing gel electrophoresis in gradient. Probes specific for the cluster Genus- and were designed to bind to sequences within the region amplified by these primers. Specific sequence of all b-subgroup of the oxidizing ammonia could not be identified, but probes specific for Nitrosomonas Nitrosospira and were designed. The presence of the Nitrosomonas cluster and Nitrosospira clusters were confirmed by denaturing electrophoresis banding patterns on gradient gel, but the results were ambiguous due to the overlapping of banding patterns. Compared to the internal control sensor signals 3 sensors Nitrosospira group decreased significantly with decreasing $\mathrm{pH}$ in the range of 06/05 to 03/08, while Division 2 Nitrosospira hybridization signal increases with acidity of the ground. At pH 5.4, cluster munitions Nitrosospira 4 signals were more, the lower upper and lower values, while the Nitrosomonas group of signals does not vary significantly with $\mathrm{pH}$. Different sets of primers for PCR amplification of the 16S rDNA sequences of the soil used in both studies and similar results demonstrate that PCR is unlikely to be an important factor. These results reveal the value of DGGE and testing for rapid analysis of b-subgroup protéobactériens communities, "oxidizing ammonia, pH differences indicates related capitals Nitrosospira populations, and shows that the cluster may have Nitrosospira importance for the ammonia-oxidant in acidic soils.
\end{abstract}

Keywords: Ammonia oxidizing bacteria, DGGE, PCR, Nitrosospira, Nitrosomonas.

\section{INTRODUCTION}

Effluents discharged from wastewater treatment plants (WWTPs) into rivers may have detrimental environmental consequences, as they are a source of high levels of nutrients, organic matter, and bacteria (Kowalchuk, 2001). The Amla Khadi is greatly affected by the discharge of the effluents from the waste water treatment plant, which treats the wastewater from 6.5 million resident equivalents. These effluents contain huge quantity s of ammonium $\left(\mathrm{NH}_{4}+\right)$, which is completely oxidized into nitrite $\left(\mathrm{NO}_{2}^{-}\right)$and subsequently converted to nitrate $\left(\mathrm{NO}_{3}^{-}\right)$by the nitrification route. Autotrophic nitrification is achieveed in two stages by two distinct groups of bacteria: nitrite oxidizers and ammonia oxidizers. Ammonia oxidation due to chemolithotrophic ammonia-oxidizing bacteria is the first and often the rate limiting step of nitrification; it is essential for the removal of nitrogen from the environment (Eichner, 1999). Aerobic autotrophic ammonia oxidizing bacteria are found within two phylogenetic groups based on comparative analyses of 16S rRNA sequences (Torsvik, 2002). One group comprises strains of Nitrosospira and Nitrosomonas spp. inside the class of BProteobacteria, and the additional contains Nitrosococcus spp. and Nitrosococcus spp. within the class of gamma-Proteobacteria. All ß-proteobacterial ammonia oxidizers belong to a phylogenetically coherent group, within which all the organisms have the same basic physiology as far as we know. A continually expanding database of ammonia oxidizing bacteria $16 \mathrm{~S}$ ribosomal DNA gene sequences has produced descriptions of distinct lineages and clusters within the genera Nitrosomonas and Nitrosospira of the B-Proteobacteria (Torsvik, 2002). Pure culture representatives have been isolated except for Nitrosospira and Nitrosomonas, where only clone sequences are accessible. A number of experimental work suggest that there are physiological and ecological differences between the different ammonia oxidizing bacteria genera and lineages and that environmental factors such as $\mathrm{pH}$, salinity and concentrations of ammonia and suspended particulate matter select for certain species of ammonia oxidizing bacteria (Kim, 2000). These external factors, which include the impact of waste 


\section{Maulin P Shah}

water treatment plant effluents, may therefore influence the range of ammonia oxidizing bacteria diversity and consequently the structure and function of the ammonia-oxidizing community. Most research has focused on ammonia oxidizing bacteria community composition in waste water treatment plant activated sludge (Rowan, 2003). Only a few studies on ammonia oxidizing bacteria diversity in freshwater environments or estuaries are available (Rowan, 2003). The application of molecular techniques, in particular analysis of $16 \mathrm{~S}$ rRNA genes, provides new opportunities for the assessment of ammonia-oxidizing populations in aquatic dregss. Phylogenetic investigation of 16S rRNA genes of pure and mixed cultures places ammonia oxidizing bacteria in three groups. Molecular analysis of ammonia oxidizer 16S rDNA fragments; amplified from environmental nucleic acid by DGGE characterizes community organization, facilitates quick analysis of clone libraries and, through excision, reamplification, and sequencing of bands provides information on species composition. This approach has reveald differences in populations of Nitrosomonas-like -proteobacterial ammonia oxidizing bacteria associated with polluted marine fish farm sediments (McCaig, 1999) and marine aggregates (Phillips, 1999) and domination by Nitrosospira-like organisms in Arctic Ocean waters (Bano, 2000). Analysis of $16 \mathrm{~S}$ rDNA does not discriminate between metabolically active and quiescent cells. Investigation of environmental 16S rRNA, using reverse transcription-PCR, provides greater sensitivity, because of the elevated intention copy number, and may designate which members of the community are further metabolically active, if energetic cells restrain larger numbers of ribosomes (Nold, 2000). The main purpose of this study was to design and test oligonucleotide probes capable of distinguishing the subgroups within the different clusters of b-subgroup ammonia oxidizers from each other and then to use these probes to identify bands separated by DGGE analysis of PCR products generated from soil with ammonia oxidizer-specific primers.

\section{Materials \& Methods}

\section{Collection and Treatment of Sample}

Five to 10 liters of water were taken for molecular, biochemical and chemical analysis and were brought to the laboratory within two to three hours. Ammonia, nitrite and nitrate are determined by spectrophotometry; The concentrations of ammonium and nitrite is determined by the order of Slawyck and MacIsaac (1972), and nitrate levels were determined after reduction of nitrite Cd (Avrahami, 2003). Suspended solids are weighed on GF / F filters were dried at $450{ }^{\circ}$ C. potential nitrification activities were determined by two methods immediately after sampling. For molecular analysis, from 150 to $250 \mathrm{ml}$ of water, filtered on nitrocellulose filters $0.22-\mathrm{mu} \mathrm{m}$ pore size in triplicate and the filters were frozen $\left(-20^{\circ} \mathrm{C}\right)$ to yield DNA. Bacterial samples for wastewater and sewage are collected by centrifugation of the samples of $1.5 \mathrm{ml}$ of $10,000 \mathrm{x}$ g for 30 minutes. Cell pellets were washed once with phosphate buffered saline (93 mM Na2HPO4, NaH2PO4 7 mmol) before being resuspended in $50 \mathrm{ul}$ of sterile distilled water, and frozen at $-20^{\circ} \mathrm{C}$ cell suspensions were subjected to three cycles of freeze-thaw and used for PCR amplification of the whole cells (Ovreas, 1997).

\section{Nucleic Acid Extraction}

Nucleic acid was extracted from the filters by the method of ball - a list of quick spin kit for DNA soil in accordance with manufacturer's instructions. The DNA extracts were stored at $-20{ }^{\circ} \mathrm{C}$ until purification column and ethanol precipitation of the Sephadex G-200 DNA was quantitated by comparison of the undiluted sample and a number of known concentrations of DNA on an agarose gel stained with ethidium bromide. To win the PCR amplicons appropriate 10- to 100- raw dilutions of the nucleic acids were used as templates for subsequent PCR. The nucleic acid was visualized by UV transillumination.

The digital images of the gels were obtained with a charge-coupled device camera controlled by Quantity One software.

\section{Oligonucleotide Probe Design}

Oligonucleotide primers were generated manually by means of a full-length $16 \mathrm{~S}$ rDNA sequences, and the use of the probe design tool WRA and the game programs in the probe (Table 1). The specificity of oligonucleotide probes was demonstrated in the BLAST (Altschul, 1990), and CHECK_PROBE (Maidak, 1999). The probes were labeled at the 5 'end of the indocarbocyanine dye (Cy-3) and / or the fluorescein. The specificity of the primers designed to amplify the template 
assigned to the spacers ammonia oxidizing bacteria are known $16 \mathrm{~S}$ rDNA clones or pure bacterial cultures. Increases by stages of annealing temperatures in the range of $46-60{ }^{\circ} \mathrm{C}$, was used to determine the optimum temperature for primer annealing. Ammonia oxidizing bacteria in general by PCR using DNA derived from biomass in the presence of ammonia oxidizing bacteria in the nitrification of the screen using the two oligonucleotides disclosed CTO189f (Kowalchuk, 1997), and NITROSO4Er (Hovanec, 1996), their annealing temperature of $57^{\circ} \mathrm{C}$ for thirty cycles. The samples were also analyzed for the presence of specific strains of ammonia oxidizing bacteria using the primers developed in the present study. Nitrosomonas ammonia oxidizing bacteria, a marina, and discovered NSMR71f and NSMR74r (54 ${ }^{\circ} \mathrm{C}$ annealing, 35 cycles), Nitrosospira as AOB discovered NSMR32f and NSMR33r (56 ${ }^{\circ} \mathrm{C}, 35$ cycles), Nitrosomonas europaea when AOB discovered NSMR52f and NSMR53r ( $56^{\circ} \mathrm{C}, 35$ cycles), and the ammonia oxidizing bacteria and Nitrosococcus mobilis'e detected NMOB1f NMOB1r $\left(56^{\circ} \mathrm{C}, 30\right.$ cycles) (table 1$)$

Table 1. The PCR sequencing primers and FISH oligonucleotides

\begin{tabular}{|l|l|l|l|}
\hline Primer/Probe & Sequence (5'-3') & $\begin{array}{l}\text { Annealing } \\
\text { temperature } \\
\left(\mathbf{(}^{\mathbf{C}}\right)\end{array}$ & References \\
\hline CTO189f & GGA GRA AAG YAG GGG ATC G & 57 & Kolwalchuk, 1997 \\
\hline NITROSO4Er & CAC TCT AGC YTT GTA GTT TC & 57 & Kolwalchuk, 1997 \\
\hline NSMR71f & CGG AAC GTA TCC AGA AGA & 54 & Kolwalchuk, 1997 \\
\hline NSMR74r & ATC TCT AGA AAA TTC GCT & 54 & This study \\
\hline NSMR32f & ATC GGA ACG TAT CTT CG & 56 & This study \\
\hline NSMR33r & CCA CCT CTC RGC GGG C & 56 & This study \\
\hline NSMR52f & TCA GAA AGA AAG AAT CAT G & 56 & This study \\
\hline NSMR53r & GTC TCC AYT AGA TTC CAA G & 56 & This study \\
\hline NMOB1f & GTT GGG AAG AAA CGA TTR CA & 56 & This study \\
\hline NMOB1r & CAC TTT TAT GTC TCC GTA AAA & 56 & This study \\
\hline EUB338 & GCT GCC TCC CGT AGG AGT & & This study \\
\hline NITROSO4E & CAC TCT AGC YTT GTA GTT TC & & This study \\
\hline Nso190 & CGA TCC CCT GCT TTT CTC C & & This study \\
\hline NSMR76 & CCC CCC TCT TCT GGA TAC & & This study \\
\hline NSMR34 & TCC CCC ACT CGA AGA TAC G & & This study \\
\hline $\begin{array}{l}\text { S-G-Ntspa-0685-a-A- } \\
\text { 22 }\end{array}$ & CAC CGG GAA TTC CGC GCT CCT C & & This study \\
\hline
\end{tabular}

\section{Probe Hybridization Analysis}

The polymerase chain reaction products were subjected to DGGE primers recovered according to the protocol CTO Muyzer et al. (1996), adapted to Kowalchuk et al. (1997) Study of ammonia-oxidizing bacteria. Gels containing 38-50\% gradient denaturing chemicals $100 \%$ is defined as denaturing $7 \mathrm{M}$ urea and $40 \%$ formamide. DNA was visualized by ethidium bromide following by UV transillumination, and the gel was recorded using 'Imager system. Of DNA in polyacrylamide gels were transferred to Hybond-N. Nucleic acid transfer membranes Transblot SD card Muyzer by others. (1996). After transplantation, the DNA is denatured through Whatman $3 \mathrm{~mm}$ filter paper, soaked in 0.4 $\mathrm{M} \mathrm{NaOH} ; 0.6 \mathrm{M} \mathrm{NaCl}$, and neutralized with $1 \mathrm{M} \mathrm{NaCl}$ in the same way; $0.5 \mathrm{M}$ Tris buffer $(\mathrm{pH} 8)$. Membranes were sealed in plastic and stored until further use $4^{3} \mathrm{C}$. Hybridization analyzes were carried out using oligonucleotide probes and hybridization conditions described by Stephen et al. (1998). Specifically CTO189f sensors NITROSO4Er, NSMR71f, NSMR74r NSMR32f and used to identify fragments of the 16S rDNA of all Nitrosospira, Nitrosomonas, ammonia oxidizers and Nitrosospira respectively. Do not attempt to quantify the intensity of radioactive signals was made during this study.

\section{Sequence Analysis of Bands Excised from Denaturing Gradient Gel Electrophoresis Gels}

The bands were excised carefully selected sequence analysis by gel electrophoresis in denaturing gradient gel with a scalpel. Only the central $50 \%$ of each strip was cut to avoid channel edges where burrs received. DNA extraction, re-amplification, and DNA sequencing are described Kowalchuk et al. (1997). Manipulation of DNA sequences was done using the program version SeqApp 1.9a169 (Kowalchuk, 1997) and a phylogenetic analysis was applied PHYLIP 5.57 (Stephen, 1998). Analyses were in accordance with the method of distance matrices P. Shah (2014) ruled the mask function and the formation of the wave data Phylogenetic tree was a neighbor joining (MP Shah, 2014). 
Phylogenetic analysis was performed with 287 positions which may be explicit to all the sequences used for the analysis. Bootstrap was made using 100 repetitions of the program SeqBoot (MP Shah, 2014). The retrieved sequences were also tested for homology to known sequences in the EMBL database using the FASTA (MP Shah, 2014). Tapes with the nucleotide sequences of the antibody designated labels in Figures 2 and 3. In addition the label characters on a tape (Figures 2 and 3) represents the difference between one pair of the numbered data sequence is based.

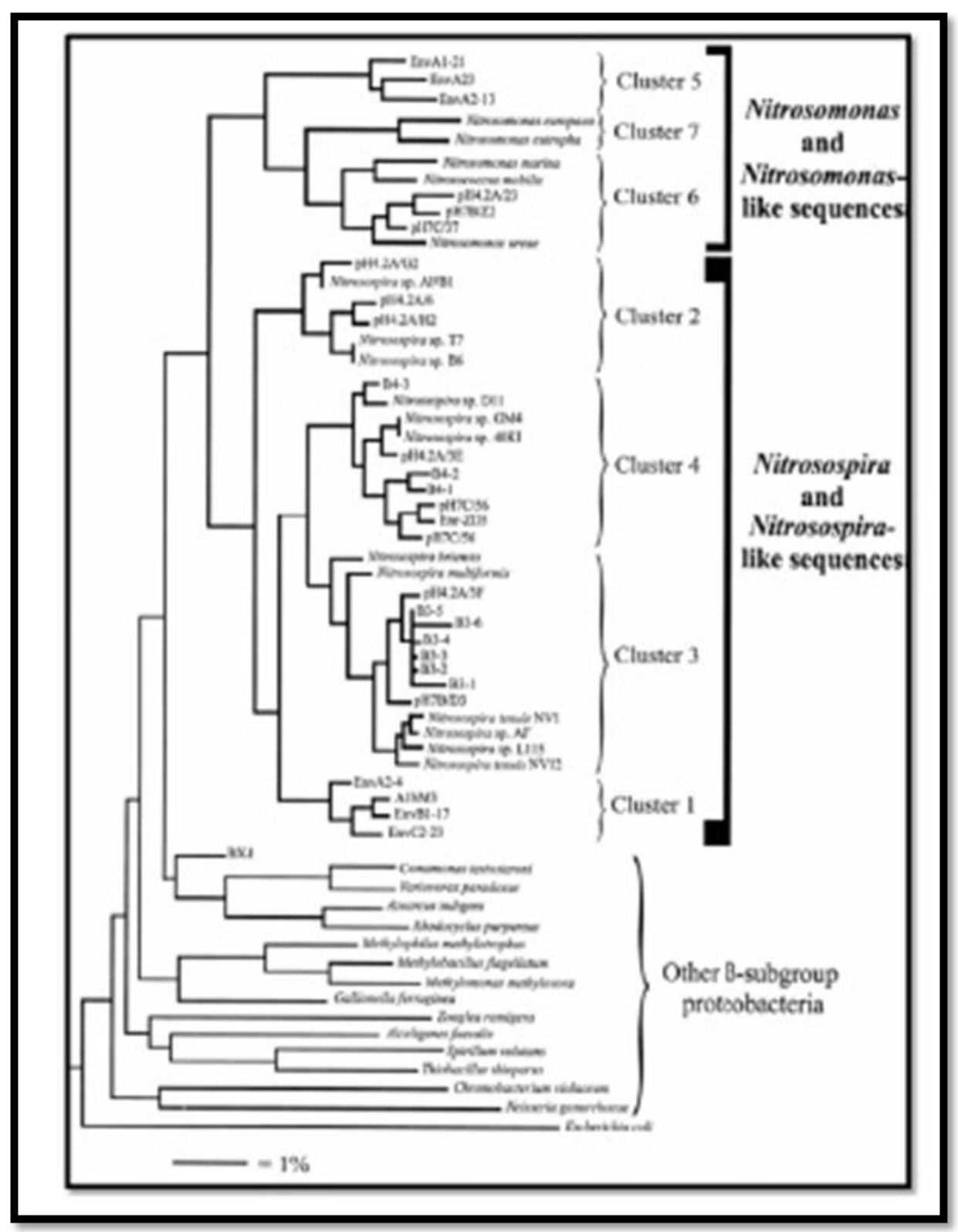

Figure 1. Neighbor joining tree based upon partial 16S Rdna sequences from L-subgroup amino oxidizers.

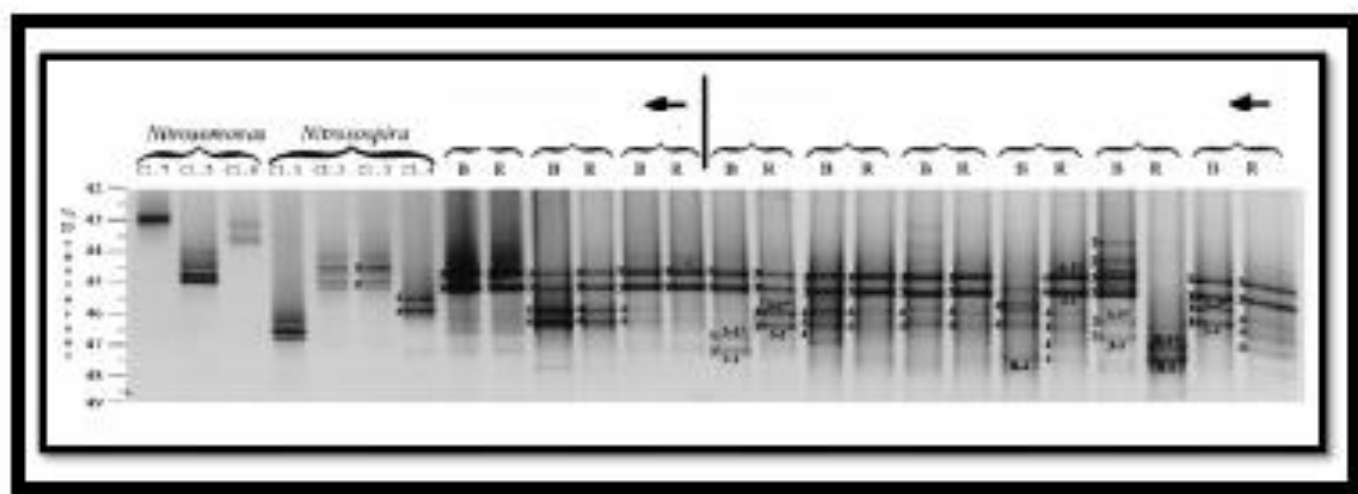

Figure 2. Denaturing Gradient Gel Electrophoresis 


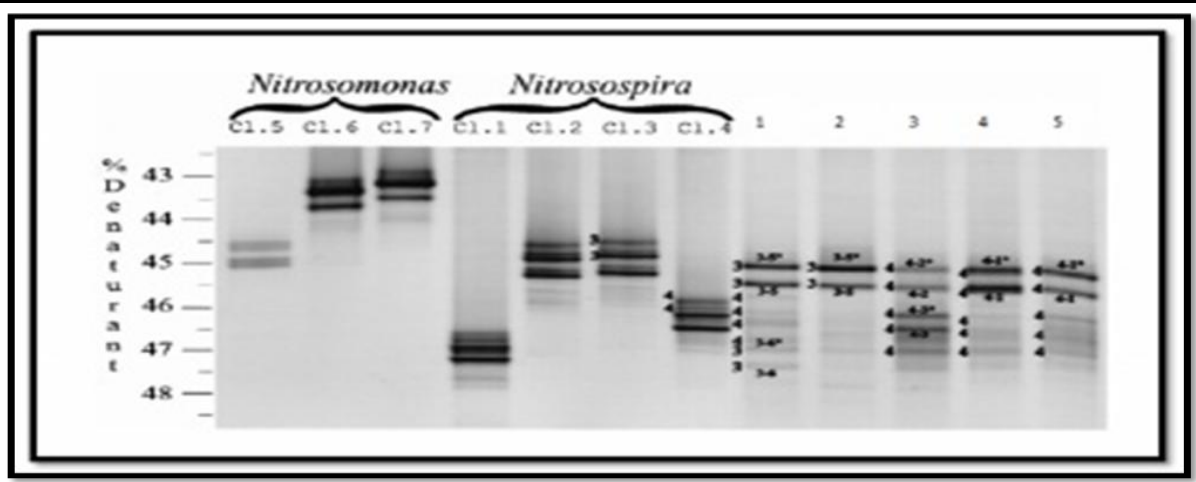

Figure 3. DGGE analysis with the same clone references used in figure 2

\section{Denaturing Gradient Gel Electrophoresis, Membrane Transfer, and Hybridization}

Polymerase chain reaction products examined a gradient denaturing gel electrophoresis the products obtained after PCR, and nested RT-specific polymerase chain reaction. $465 \mathrm{bp}$ fragment was used for the 16S rRNA gene and lasted for the GC clamp is composed of 36 bp (Sheffield, 1989), introduced during the polymerase chain reaction. Electrophoresis in a denaturing gradient gel was performed using the protocol D-Gene system Muyzer et al. (26) is adapted for analysis of the subgroup B of oxidizing ammonia protéobactériens (19). DNA fragments of known oxidizing ammonia ammunition views of the sequence (Fig. 1) (42) were also subjected to gel electrophoresis on denaturing gradient gel electrophoresis (denaturing 38 to 50\%) as controls for hybridization assays later. Of the nucleic acid stained by ethidium bromide and rinsed twice with deionized water before UV transillumination. The DNA was transferred to Hybond-N1 polyacrylamide gels to membranes for the transfer of nucleic acid using a Bio-Rad Model transblotter SD semi-dry, as described Muyzer et al. (1996). The DNA was then denatured (DNA side down) and at the same time cross-linked to the membrane soaked in $0.4 \mathrm{M} \mathrm{NaOH}, 0.6 \mathrm{M} \mathrm{NaCl}$, the filter paper with a $3 \mathrm{~mm}$. Membranes were similarly neutralized with $\mathrm{M} \mathrm{NaCl}-0.5 \mathrm{M}$ Tris $\mathrm{HCl}(\mathrm{pH}$ 8). Hybridization analyzes were carried out using oligonucleotide probes and hybridization conditions described by Stephen et al. (1998). No list has been caught in the intensities of the hybridization signals received.

\section{Quantification of Relative Abundances of Sequence Types}

Gels were first probed b-AO233 for quantizing a signal of ammonia $\beta$-Proteobacteria total oxidant into the PCR products. Connected to the relative abundance of two or three clusters were calculated by subtracting the total hybridization. Loadss compare the two, and three groups were determined by sampling and gels NspEL206 NspEL224, a specific cluster of two and three in the case. The gels were probed with b-AO233 checks showed that both clusters were loaded at equal and the three-level (less than $0.5 \%$ of the other). The differences in the efficiency of binding and other factors among a cluster of two and three probes was determined by comparing hybridization signals of appropriate controls, consisting of diplomats from the clones. Consequential correction factors used in the calculation of the relative hybridization signal samples cluster two and three signals. Finally, these values were expressed as a percentage of the cluster associated with the two or three different frequency signals for each group to give a percentage of the total ammonia oxidizer indication. All test analyzes were performed on two sets of a sovereign, soil samples, and the results are presented as the means of duplicates.

\section{Results}

\section{Probe Design}

The specificity of the probe, both pragmatic and controlled search database is shown in Table 1 . The natural communities were analyzed by denaturing gradient gel electrophoresis gel, probing the product obtained by polymerase chain reaction primers specific for $\beta$-Proteobacteria oxidizing ammonia (Kowalchuk, 1997). The probe was chosen for their ability to distinguish between different groups of ammonia oxidants belong. Some also have the ability to distinguish between non-oxidizing ammonia, ammonia-oxidizing bacteria. They bear at least one or the two current differences between the non-target sequences from the database (Table 1). None of the target site probe, which is specific for all of the ammonia oxidizing $\beta$-sub-groups, and which is able to distinguish these organisms from all other $\beta$-Proteobacteria were found in the region amplified. NMOB1f. The probe did not reveal the full specificity of SS ammonia oxidizers search restrictions to existing databases. However, all non- 
target strains, similar sequences were highly destabilizing discrepancies in a polymerase chain reaction primer Polymerase chain reaction used to produce the CTO products for denaturing gradient gel electrophoresis. NMOB1f probe was used to normalize the study of radioactive products resulting from amplification with primers CTO measured relative each strip isolated denatured gradient gel electrophoresis.

\section{Nitrosospira Gene}

Most ground clone partial sequences were recovered from the three groups within the Nitrosospira group (Figure 1, cluster 2, 3, and 4). Cluster 3 also contained most of the partial sequences culture Nitrosospira spp. In the resolution of our analysis, it does not appear that the composition of the analogous Nitrosospira cluster was entirely determined by soil $\mathrm{pH}$ from which the library was constructed. Each of the three poles of the earth contained sequences each $\mathrm{pH}$, and in each group there are examples where the same partial sequence was recovered from two floors. However, the group 2 contained most of the sequences of the soil $\mathrm{pH} 4.2$ (16 of 20 sequences), and group 3 contained a higher proportion of sequences (10 of 12 sequences) from the soil $\mathrm{pH} 7.0$. There is an evidence of our analyses that marine clones Nitrosospira - as are distinct from the sequences of clones soil Nitrosospira - like. All marine clones formed a separate group (Fig. 1, Group 1) whose relationship to gender Nitrosospira was strongly supported by the analysis of three longer sequences (Fig. 1). In this clade sailor, there were sequences of the three sediment samples, and even sometimes partial sequence was recovered from different samples.

\section{Genus Nitrosomonas}

Partial sequences clustering with cultivated species Nitrosomonas were detected in all samples. Two floor clones from neutral and a ground floor acid formed a strongly supported clade (Fig. 1) in a radiation (Fig. 1, the group 6), which further comprises sequences from of marine samples. Sequencing Fulllength acidic soil clone (designated pH4.2 / 23; See the legend of Figure 1 for an explanation of clones' designations) supports its distinct status within the Nitrosomonas clade (Figure 2). The nine sequences Nitrosomonas -as were remaining marine sediments. Six of them from the most polluted site (A) and the site showing through impact (B) formed a new group (Fig. 1, the group 5 ) which does not contain sequences from the site apparently unpolluted C. Evidence from partial sequences gave a weakly supported clade relationship between 5 and gender Nitrosospira. However, analysis of longer sequences of two clones strongly supported the accession of the Nitrosomonas clade with moderate support for a relationship Nitrosomonas sp. NM63 and cloned soil pH4.2A / 23.

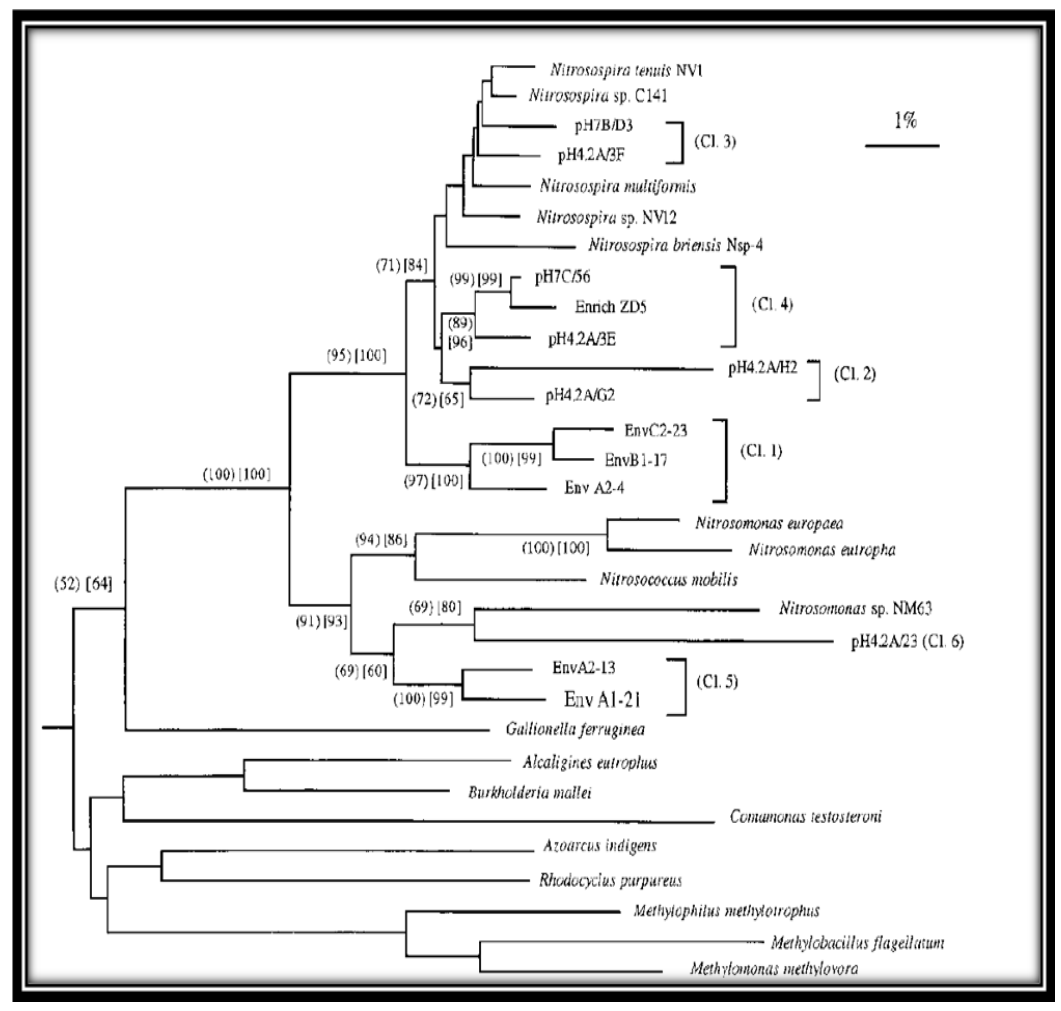

Figure 4. Neighbor-joining tree showing relationships between environmental sequences and reference $\beta$ proteobacteria based on 1,099 bases of aligned 16S rDNA sequences. 


\section{Examination of Environmental Samples by Denaturing Gradient Gel Electrophoresis and Probing}

Polymerase chain reaction amplification, the GC clamp incorporates, for denaturing gradient electrophoresis gel analysis, were obtained. Polyacrylamide gel was stained with ethidium bromide, and 5 illustrate Autoradiographs were obtained by-the use of oligonucleotide probes specific for the B subgroup of ammonia oxidizers (Table 1). Southern hybridization of the probe b-AMO233 (Fig. 5B), which is designed to detect all of the B-oxidants subgroup of the Proteobacteria of ammonia leads to bind to all of the bands present on the gel was stained with éthidium- bromide (Fig. 5A). Fingerprints line. $5 \mathrm{C}$ and $\mathrm{D}$, one of the clusters probed with the oligonucleotide probe, and the marine sea Nitrosospira Nitrosomonas spp. (6b group only) is shown in accordance with the fact that these organisms were present in all samples of sludge. The blot shown in Fig. 5E, which shows the result of hybridization with a probe specific for any Nitrosomonas clusters (Cluster 5 to 7), an additional doublet band was detected, the signal intensity of these channels corresponds to a very contaminated sediments (lanes 1 and 2) to a less intense signal at $20 \mathrm{~m}$ Station (lanes 3 and 4), and the signal available at a temperature of $40 \mathrm{~m}$ (lanes 5 and 6). Although the intensity of the twin declined compared with less pollutants, it seems that a corresponding increase in the intensity of the band less than one cluster sequences Nitrosospira stations 20 and $40 \mathrm{~m}$. This was confirmed by a quantitative tape density (Fig. 6), which shows the relative contribution of each of the sub band signals on an estimated total of hybridization probe $\beta$-AO233 DGGE is obtained for each sampling station. The band did not hybridize with the twins is not $6 \mathrm{~b}$ (Fig. 5D) or cluster oligonucleotide probes 7 , but in cooperation with the control clone migrated Romance, Nitrosomonas sea clones. Based on the sequence of current information, it is possible to design specific oligonucleotide probes ammunition 5; Thus, identity confirmed by sequence analysis of the upper and lower bands. Both groups produced identical sequences of $422 \mathrm{bp}$ and as expected, it appears that the decline of the PCR reverse primer is responsible for the observed effect. A similar explanation may be applied for the control group of the first track number of banding Cluster 5 DGGE sequences indicate identity to $0.99 \%$ (1 bp shift) the partial sequence of the clone EnvA2-13, which is obtained in the same samples by using different primers. Phylogenetic analysis shows clearly that the tape sequences are grouped in a cluster DGGE nitrosomonas 5 (100\% of bootstrap replicates), as shown in Fig. 1. Quantifying the relative abundance of different groups was performed densitometry. Figure 6 shows the relative intensities of the band obtained when the probe b-AO223, which detects all of the clusters protéobactériens oxidants $\mathrm{B}$ subgroup of ammonia was used. Not Nitrosospira one of the most common of a group of three sites, including 53-83\% of the total signal in each channel. This is consistent with the initial detection of Stephen et al. (1998), the Group 1 Nitrosospira spp. have been found, and is a potentially rich source of the marine environment. The relative amount of signals of ammunition belts 5 , the probe was determined using a b-AO223 (Fig. 5B), was significantly higher than the site $\mathrm{B}$, site $\mathrm{C}$, or $(\mathrm{P}=0.01)$. Although Fig. 5B-E, seems to be an increase in both groups 1 and 6, with the increase in distance from the fish cage, with no significant difference in the relative abundance of these groups in one of the sites was observed. Quantification of the total signal for each track gel with ethidium bromidestained and after probing the $\mathrm{B}-\mathrm{AO} 233$, showing an increase of probe hybridization with distance from the fish cages, despite the burden of the DNA bit about equal. This may be explained by non-amplification of the target sequences during PCR organism or presence in oxidizing ammonia in the contaminated sediments rDNA sequences which are not complementary to the oligonucleotides used in this study. Quantifying the relative abundance of Nitrosomonas clusters 5 and 6, when probed with probe nitrosomonas Nmo254, confirmed that a group of five signals decreased significantly compared to the total signal in each channel, increase the distance to the fish cage. It was not possible in this study; However, in order to quantitatively compare the relative abundance of the fifth group, using both the probe and the Nitrosomonas Nmo254 B AMO233. A long exposure of Nmo254 autoradiography (Fig. 5E), it was necessary to look at the 5 tape group means a representative sample of the stations B, but as a result of saturation of Group 5 and 6 controls, preventing data standardization for both probes. However, compared to the ratios for each group of samples, using both probes, indicating that the effectiveness of these two probe oligonucleotides were similar. 


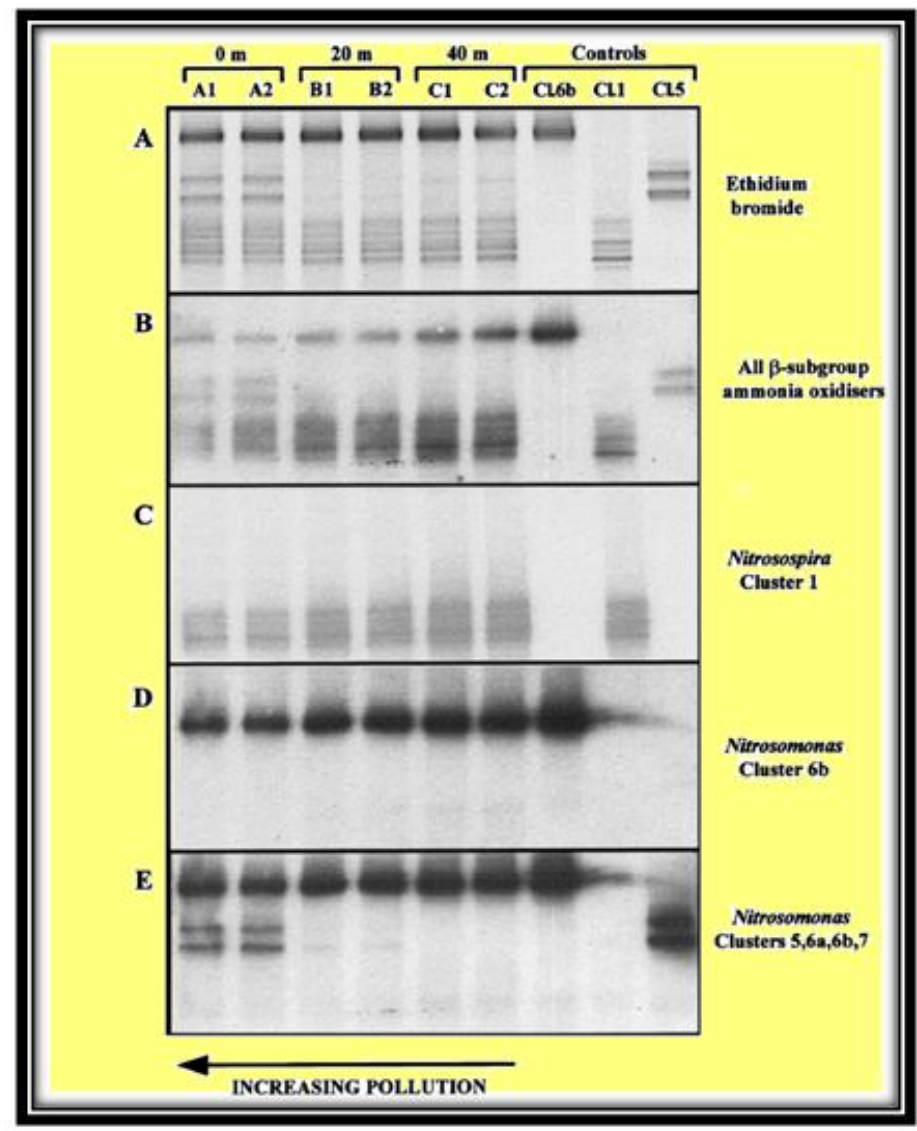

Fig 5. Denaturing Gradient Gel Analysis and of $\beta$-subgroup ammonia-oxidizing bacteria.

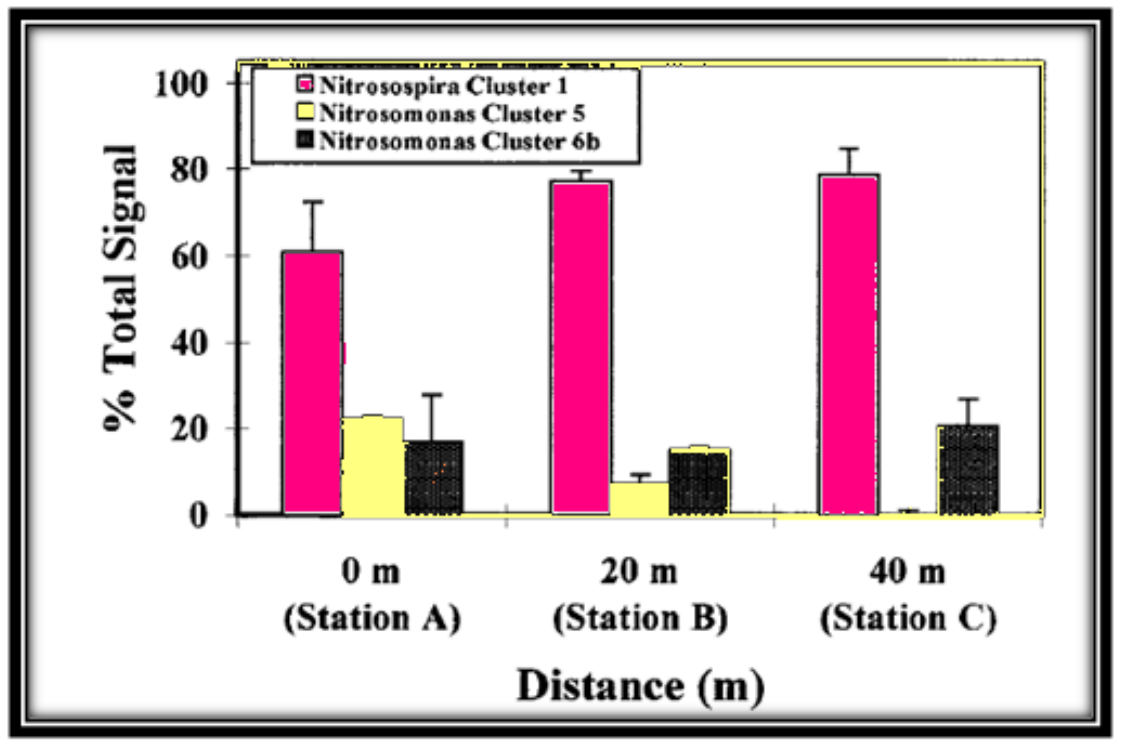

Fig 6. Relative abundance of b-subgroup ammonia-oxidizing bacteria Ammonia oxidizer clusters were resolved by DGGE, and relative band intensities were quantified by densitometry after probing with $b$-AMO233, which is specific for all b-subgroup ammonia oxidizing bacteria. Error bars represent standard errors for duplicate sediment samples.

\section{Discussion}

Definitive correlation studies with nitrification rate of nitrifying micro-organisms in natural samples are difficult. Until recently, there were few methods available to identify and quantify bacteria or groups of bacteria in specific for environmental samples without culture (Amann, 2008). Ward (2007) 
used immunofluorescence techniques to count the nitrifying bacteria, but this technique also requires the culture of the target to produce antibodies. The culture of nitrification bacteria is particularly difficult because of the low rate of growth of these bacteria and the frequent occurrence of crop contamination by heterotrophic bacteria (Voytek, 2009). The current development of the 16S rDNA sequence information $\beta$-oxidation of ammonia subgroup boosted the potential value of molecular techniques in the investigation of the ecology of this group of ecologically and economically important microorganisms. Most of the ammonia oxidizing bacteria diversity studies using 16S rDNA primers targeted through the natural environment, such as agricultural, marine sediments (Stephen et al., 1998), and the lakes of water column (Ward et al., 2000) or the center hole of the Arctic Ocean (Bano and Hollibaugh 2000). Alternatively, the study of stress distribution in the environment groups is the use of ammonia oxidizing specific oligonucleotide probes, but previous reports have been limited to use of sequence information cultures of pure design and test probes. Some of the work has focused on the sediment environment change systems such as farms (McCaig et al., 1999), but little work has been done so far with artificial systems such as water treatment plants waste, reactor and wetlands, which are highly active nitrogen regimes. Phylogenetic analysis showed the division boxidizing ammonia subgroup number of clusters or related sequences. The study is the abundance of sequence achieved by polymerase chain reaction amplification in environmental samples using primers specific for ammonia oxidizer It also proposes the distribution of the different groups Linked to Environmental Factors. In addition, the methods used so far are mainly based on the polymerase chain reaction amplification, cloning and sequencing are thereafter. However, this primer Gives specificity when used as a group, such as the polymerase chain reaction amplification primers and specific probes are unreliable specific oxidizing ammonia. Here we used the FISH probe base Nso190 Because of its high specificity for $\beta$-HCA, and because it allows a proper size and DGGE PCR fragment was generated. In one primer Eub787F eubacteria PCR fragment of about $500 \mathrm{bp}$ can be obtained, which is informative enough for phylogenetic conclusions are drawn (Stackebrandt and Rainey, 1995). It was well known that the performance of waste water treatment plant is primarily determined by the structure and activity of microbial communities (Cook, 2006). In these studies, is the degeneration of the primer or the size of the amplified fragment does not allow using the high resolution of the fingerprint and the DGGE method, such as regular monitoring of the populations of ammonia oxidizing bacteria is not possible. Contrary to current data, the rDNA sequences of primers of CTO (Kowalchuk, 1997) are specific for the B-oxidants, all subgroups of ammonia. However, our understanding of the relationship between the structure and function of the microbial community is still limited and even contradictory. Is designed to display this function bioreactor as substrate for the removal changed by concomitant changes in the structure of microbial community in response to an alarm (Lapara, 2000) or during the acclimatization (Lapara and Ghosh, 2006), and the function Stable relates to the structure of a stable communities (Ayala-Del -R, 2004). It is encouraging that the analysis of new environments to identify additional sequences which hybridize Nso190. This anxiety need for the creation of clone libraries to launch a new research environments, and the use of PCR primers of low specificity for the detection of related groups, but did not characterize (McCaig, 1994). On the other hand, it was found that the structure of the microbial biomass in the bioreactor has fluctuated functionally stable (Fernandez, 2000), which may be due to the phenomenon that the same physiological function can be divided among the various micro-organisms in particular of functional redundancy of the microbial community (Franklin and Mills, 2006). In addition to the functional stability showed less stable community in response to the disturbance of glucose (Fernandez, 2000). Further analysis of the target region used in this study, comprises 318 bases 168-510 of Escherichia coli in a homologue position, does not give any specific sequence unique oxidizing ammonia. It was possible, however, to design the probe, which seems to be specific to Nitrosospira group. Nitrosomonas Nitrosomonas Nso190 probe was specific for all sequences achievees our study site, but do not intersect ureae Nitrosomonas Nitrosomonas communis, and the recently-sequenced Pommerening Roser et al. (1996). This may be due to changes in the functionally significant populations but quantitatively smaller, which can escape the detection of the analysis of the structure oriented to the general public. Denaturing gradient gel electrophoresis technique used in the page Poll of this study included a preliminary amplification primers specific for CTO oxidizing ammonia before using probes. A limitation inherent in any electrophoresis technique is also applicable to a denaturing gradient gel electrophoresis. Denaturing gradient gel electrophoresis Engender number of bands may not accurately reflect the number of different sequences present in the mixture. Undoubtedly, the nucleic acid of bacteria, which are present in the environment, may gain access to a relatively small 


\section{Maulin P Shah}

number of amplification by polymerase chain reaction. In addition, denaturing gradient gel electrophoresis band may represent a total of some species, or a lot of bands can cause the same bacteria as degenerate (Se'bilo, 2003), the formation of heteroduplexes or more heterogeneous rRNA operon (Deer, 2001). This confirms the specificity of PCR primers and reaction conditions described in a variety of environments. In this situation, the probes specific for a cluster analysis applied to a pair of primers with PCR products generated from the CTO oxidative subgroup b must be faithful clade ammonia. This study also provides information needed to use the hybridization probe for each cluster. Hybridization Td values calculated from the elution profiles of some of the target and probe sequences were able to distinguish between target and nontarget ammonia oxidizer known sequences. Another advantage of using a primer Nso190 is that its sensitivity is greater than that of other targeted primers 16SrDNA. Our analysis of the PCR products was directly obtained from the genomic DNA. However, previous studies have reported the need for most of the $\beta$-HCA nested PCR specific primers as previously described (Kowalchuk, 1997). Effect on the growth of heterotrophic bacteria in the percentage of the ammonia oxidizing bacteria and nitrite oxidizing bacteria, nitrite oxidizing bacteria, it is evident considering the number of samples of the biomass before and after inoculation. All of ammonia oxidizing bacteria identified putative clone libraries were part of the class Proteobacteria. Phylogenesis of four strains of ammonia oxidizing bacteria recovered 16S rRNA Phylogenetic tree biomass in this study, as shown in Fig. The protocol used for the extraction of DNA (Regan, 2002) should also be considered, because it leads to models without Humiid - addicts, which can be partly explained by the performance of our primers. DGGE followed by polymerase chain reaction products Poll professional groups confirmed these decisions. 3, the signals do not have probes rose steadily soil $\mathrm{pH}$ decreased from 6.4 to 3.6, while the Group 2 decreased by the probes in this range. Both of these studies have also shown similar levels of Class 6 at all $\mathrm{pH}$ values of the soils studied and said Nitrosospira dedicated cluster 2 and clusters nitrosomonas 3, 4, 5, 6b, and 7 missing in this environment, or below the detection limits of this approach are the time of sampling. Confirmation that there are strains of the overall oxidation of ammonia and experts stressed the need for studies of the psychology of these groups. Several clusters of 2 strains are available in pure cultures and pure cultures of the two clusters Nitrosospira 3 and Nitrosospira sp. B6 and T7 strains are newly obtained (Utåker, 2004). At present, nothing is known about the physiological differences, which can lead to the selection as a function of $\mathrm{pH}$, but our results indicate that it may be important not Nitrosospira 2 genotype in acid soils and physiological studies of representatives of this group is required. Phylogenetic analysis of the topology of the tree parsimony bootstrap analysis, and analysis of the similarity matrix shows that like ammonia oxidizing bacteria Nitrosospira represent a single clade characterized briensis Nitrosospira multiforme Nitrosospira and Nitrosospira tenuis (Fig. 1). The ammonia oxidizing bacteria tenuis increased Nitrosospira best to maintain a high concentration of ammonia reactors. However peripherals Nitrosospira tenuis ammonia oxidizing bacteria such as nitrification were able to accelerate the addition of new aquariums. However, Nitrosospira tenuis ammonia oxidizing bacteria are not able to identify the majority of the biomass, samples of PCR or FISH aquarium for several weeks after the addition. So it seems that the ammonia oxidizing bacteria Nitrosomonas marina such as the ability to compete Nitrosospira tenuis bacteria - an oxidizing agent such as ammonia in the environment at low concentrations of ammonia in the aquarium. Map genusand specific probes and their use in a cluster with a permutation of DNA DGGE analysis of PCR amplification products obtained directly from the ground to provide powerful technique for quick and quantitative investigation of the structure of natural populations of ammonia oxidizer environments. The technique has shown clear links between genotype and soil ecotype is maintained in the range of $\mathrm{pH}$ values for 36 years, identifying the general and candidate strains professionals. The results provide the basis for further studies on the physiological characteristics of these strains leading to differences in their distribution and their significance for nitrification rates and persistence of ammonia oxidizers in natural environments.

\section{REFERENCES}

[1] Altschul SF, W Gish, W Miller, EW Myers, DJ Lipman (1990). Basic local alignment search tool. J. Mol. Biol. 215:403-410

[2] Amann, R. I., W. Ludwig, and K.-H. Schleifer. 2008. Phylogenetic identifi- cation and in situ detection of individual microbial cells without cultivation. Microbiol. Rev. 59:143-169

[3] Avrahami S, W Liesack, R Conrad (2003). Effects of temperature and fertilizer on activity and community structure of soil ammonia oxidizers. Environ. Microbiol. 5: 691-705. 
[4] Ayala-Del-Rio, H.L., Callister, S.J., Criddle, C.S., Tiedje, J.M., 2004. Correspondence between community structure and function during succession in phenol- and phenol-plus-trichloroethenefed sequencing batch reactors. Appl. Environ. Microbiol. 70, 4950-4960

[5] Bano N, Hollibaugh JT (2000) Diversity and distribution of DNA sequences with affinity to ammonia-oxidizing bacteria of the $\beta$ - subdivision of the class Proteobacteria in the Arctic Ocean. Appl Environ Microbiol 66:1960-1969

[6] Bano N, J. Hollibaugh (2000). Diversity and distribution of DNA sequences with affinity to ammonia-oxidizing bacteria of the subdivision of the class Proteobacteria in the Arctic Ocean. Appl. Environ. Microbiol. 66: 1960-1969.

[7] Cook, K.L., Garland, J.L., Layton, A.C., Dionisi, H.M., Levine, L.H., Sayler, G.S., 2006. Effect of microbial species richness on community stability and community function in a model plantbased wastewater processing system. Microb. Ecol. 52, 725-737

[8] Daims, H., J. L. Nielsen, P. H. Nielsen, K.-H. Schleifer, and M. Wagner. 2001. In situ characterization of Nitrospira-like nitriteoxidizing bacteria active in wastewater treatment plants. Appl. Environ. Microbiol. 67: 5273-5284.

[9] Eichner CA, RW Erb, KN Timmis, I Wagner-Dobler (1999). Thermal gradient gel electrophoresis analysis of bioprotection from pollut-ant shocks in the activated sludge microbial community. Appl. Environ. Microbiol. 65: 102-109

[10] Fernandez, A.S., Hashsham, S.A., Dollhopf, S.L., Raskin, L., Glagoleva, O., Dazzo, F.B., et al., 2000. Flexible community structure correlates with stable community function in methanogenic bioreactor communities perturbed by glucose. Appl. Environ. Microbiol. 66, 4058-4067.

[11] Franklin, R.B., Mills, A.L., 2006. Structural and functional responses of a sewage microbial community to dilution-induced reductions in diversity. Microb. Ecol. 52, 280-288

[12] Hovanec TA, EF DeLong (1996). Comparative analysis of nitrifying bacteria associated with freshwater and marine aquaria. Appl. Environ. Microbiol. 62:2888-2896

[13] Kim IS, VN Ivanov (2000). Detection of nitrifying bacteria in activated sludge by fluorescent in situ hybridization and fluorescence spectrometry. World J. Microbiol. Biotechnol. 16: 425-430.

[14] Kowalchuk GA, JR Stephen (2001). Ammonia-oxidizing bacteria: a model for molecular microbial ecology. Annu. Rev. Microbiol. 55: 485-529

[15] Kowalchuk GA, JR Stephen, W de Boer, JI Prosser, TM Embley, JW Wolderdorp (1997). Analysis of ammonia-oxidizing bacteria of the subdivision of the class Proteobacteria in coastal sand dunes by denaturing gradient gel electrophoresis and sequencing of PCR-amplified 16S ribosomal DNA fragments. Appl. Environ. Microbiol. 63:1489-1497.

[16] LaPara, T.M., Ghosh, S., 2006. Population dynamics of the ammonia-oxidizing bacteria in a fullscale municipal wastewater treatment facility. Environ. Eng. Sci. 23, 309-319. LaPara, T.M., Konopka, A., Nakatsu, C.H., Alleman, J.E., 2000. Effects of elevated temperature on bacterial community structure and function in bioreactors treating a synthetic wastewater. J. Ind. Microbiol. Biotech. 24, 140-145.

[17] LaPara, T.M., Konopka, A., Nakatsu, C.H., Alleman, J.E., 2000. Effects of elevated temperature on bacterial community structure and function in bioreactors treating a synthetic wastewater. J. Ind. Microbiol. Biotech. 24, 140-145

[18] Maidak BL, JR Cole CT Parker, J GM. Garrity, N Larsen, B Li, TG Lilburn, MJ McCaughey, GJ Olsen, R Overbeek, S Pramanik, TM Schmidt, JM Tiedje, C. R. Woese. 1999. A new version of the RDP (Ribosomal Database Project). Nucleic Acids Res. 27:171-173

[19] McCaig AE, Phillips CJ, Stephen JR, Kowalchuk GA, Harvey SM, Herbert RA, Embley TM, Prosser JI (1999) Nitrogen cycling and community structure of proteobacterial $\beta$-subgroup ammonia-oxidizing bacteria within polluted marine fish farm sediments. Appl Environ Microbiol 65:213-220

[20] McCaig, A. E., C. J. Phillips, J. R. Stephen, G. A. Kowalchuk, S. M. Harvey, R. A. Herbert, T. M. Embley, and J. I. Prosser. 1999. Nitrogen cycling and community structure of proteobacterial beta-subgroup ammonia-oxidizing bacteria within polluted marine fish farm sediments. Appl. Environ. Microbiol. 65:213-220. 
[21] McCaig, A. E., T. M. Embley, and J. I. Prosser. 1994. Molecular analysis of enrichment cultures of marine ammonia oxidisers. FEMS Microbiol. Lett. 120:363-368.

[22] Muyzer G, Hottentrager S, Teske A, Wawer C (1996) Denaturing gradient gel electrophoresis of PCR-ampliced 16S rDNA. A new molecular approach to analyze the genetic diversity of mixed microbial communities. In: Molecular Microbial Ecology Manual (Akkermans, A.D.I., Van Elsas, J.D. and De Bruijn, F.J., Eds.), pp. 3.4.4.1-3.4.4.22. Kluwer, Dordrecht.

[23] Nold, S. C., J. Zhou, A. H. Devol, and J. M. Tiedje. 2000. Pacific Northwest marine sediments contain ammonia-oxidizing bacteria in the subdivision of the Proteobacteria. Appl. Environ. Microbiol. 66:4532-4535.

[24] Ovreas L, Forney L, Daae F L `et al., 1997. Distribution of bacterioplankton in meromictic lake Saelevannet, as determined by denaturing gradient gel electrophoresis of PCR-amplified gene fragments coding for 16S rRNA[J]. Appl Environ Microbiol, 63: 3367-3373.

[25] Phillips, C. J., Z. Smith, T. M. Embley, and J. I. Prosser. 1999. Phylogenetic differences between particle-associated and planktonic ammonia-oxidizing bacteria of the -subdivision of the class Proteobacteria in the northwestern Mediterranean Sea. Appl. Environ. Microbiol. 65:779-786.

[26] Pommerening-Ro"ser, A., G. Rath, and H.-P. Koops. 1996. Phylogenetic diversity within the genus Nitrosomonas. Syst. Appl. Microbiol. 19:344-351.

[27] Regan JM, Harrington GW, Noguera DR (2002) Ammonia- and nitrite-oxidizing bacterial communities in a pilot-scale chloraminated drinking water distribution system. Appl Environ Microbiol 68:73-81

[28] Rowan, A. K., J. R. Snape, D. Fearnside, M. R. Barer, T. P. Curtis, and I. M. Head. 2003. Composition and diversity of ammoniaoxidising bacterial communities in wastewater treatment reactors of different design treating identical wastewater. FEMS Microbiol. Ecol. 43: 195-206

[29] Se'bilo, M. 2003. Utilisation du trac,age isotopique naturel pour caractériser et quantifier les processus de nitrification et de de'nitrification a' l'e'chelle du re'seau hydrographique de la Seine. Ph.D. thesis. Universite' Pierre et Marie Curie, Paris, France.

[30] Shah MP (2014) Exploring the Strength of Pseudomonas putida ETL-7 in Microbial Degradation and Decolorization of Remazol Black-B. International Journal of Environmental Bioremediation and Biodegradation 2: 12-17.

[31] Shah MP (2014) Microbiological Removal of Phenol by an Application of Pseudomonas spp. ETL. An Innovative Biotechnological Approach Providing Answers to the problems of FETP. Journal of Applied and Environmental Microbiology 2: 6-11.

[32] Sheffield, V. C., D. R. Cox, and R. M. Myers. 1989. Attachment of a 40-base pair G1C-rich sequence (GC-clamp) to genomic DNA fragments by the polymerase chain reaction results in improved detection of single-base changes. Proc. Natl. Acad. Sci. USA 86:232-236.

[33] Slawyck, G., and J. J. McIsaac. 1972. Comparison of two automated ammonium methods in a region of coastal upwelling. Deep Sea Res. 19: 1-4

[34] Stackebrandt E, Rainey FA (1995) Partial and complete 16S rDNA sequences, their use in generation of $16 \mathrm{~S}$ rDNA phylogenetic trees and their implications in molecular ecological studies. In: Molecular microbial ecology manual, vol. 3.1.1. Kluwer, The Netherlands, pp 1-17

[35] Stephen JR, Kowalchuk GA, Bruns MAV, McCaig AE, Phillips CJ, et al. (1998) Analysis of beta-subgroup proteobacterial ammonia oxidizer populations in soil by denaturing gradient gel electrophoresis analysis and hierarchical phylogenetic probing Appl Environ Microbiol 64: 2958-2965

[36] Torsvik, V., and L. Ovreas. 2002. Microbial diversity and function in soil: from genes to ecosystems. Curr. Opin. Microbiol. 5: 240- 245

[37] Utåker, J. B., L. Bakken, Q. Q. Jiang, and I. F. Nes. 1996. Phylogenetic analysis of seven new isolates of ammonia-oxidising bacteria based on 16S rRNA gene sequences. Syst. Appl. Microbiol. 18:549-559.

[38] Voytek, M. A., and B. B. Ward. 2009. Detection of ammonium-oxidizing bacteria of the betasubclass of the class Proteobacteria in aquatic samples with the PCR. Appl. Environ. Microbiol. 61:1444-1450 
[39] Ward BB, Martino DP, Diaz MC, Joye SB (2000) Analysis of ammonia-oxidizing bacteria from hypersaline Mono Lake, California, on the basis of 16S rRNA sequences. Appl Environ Microbiol 66:2873-2881

[40] Ward, B. B. 1982. Oceanic distribution of ammonium-oxidizing bacteria determined by immunofluorescence. J. Mar. Res. 40:1155-1172. 